\title{
Numerical investigation of the influence of explosive welding process setup on the $\mathrm{Ti} / \mathrm{Cu}$ interlayer morphology
}

\author{
Mateusz Mojżeszko*1 (DD, Mohan Setty² (iD)
}

${ }^{1}$ AGH University of Science and Technology, al. Mickiewicza 30, 30-059 Krakow, Poland.
${ }^{2}$ Institut for Frontier Materials, Deakin University, 75 Pigdons Road, Waurn Ponds VIC 3216, Australia.

\begin{abstract}
Explosive welding is a complex process involving various phenomena influenced by a series of parameters in a noticeably short span of time which affect the morphology and eventually the quality of the weld. Therefore, this paper aims to investigate the influence of these parameters on material behavior with a series of numerical simulations based on a meshless approach. The developed model is based on the SPH (Smoothed Particle Hydrodynamics) method and is used to investigate Ti/Cu system behavior as a case study. Examples of the resulting temperatures and pressures as a function of process setup are presented within the paper. The results obtained demonstrate how weld morphology is related to the process conditions.
\end{abstract}

Keywords: explosive welding, meshless model, smoothed particle hydrodynamics.

\section{Introduction}

Laminated bi-layered products have broad development prospects and a wide variety of applications across many different fields, for example in aerospace or equipment manufacturing sectors where conventional materials properties are insufficient (Wachowski et al., 2018). The manufacturing process of these products is not only justified from fulfilling functional requirements, but also from an economical point of view. Most of the time it is sufficient to apply only a thin layer of material with elevated properties and to use a cheaper material as a base. During the course of the development of multi-layered materials, many different approaches were proposed, e.g. laser welding (Lednev et al., 2021), vibration welding (Wolf et al., 2020), electromagnetic pulse welding
(Li et al., 2021), or explosive welding presented by Akbari Mousavi and Farhadi Sartangi (2009).

In the latter case, the explosively driven impact is an extraordinarily complex process involving various phenomena interacting with each other during a concise time frame. This involves heat transfer between colliding plates, heat generation due to the friction, high pressure, or local diffusion within the interacting materials. Therefore, this process is challenging to be observed and measured during experimental analysis. Figure 1 shows the basic concept of explosive welding technology.

As seen in Figure 1, the flyer plate is propelled by the detonation of the explosive material placed at the flyer plate's top surface. The resulting force draws the two plates together under high pressure.

*Corresponding author: mmojzesz@agh.edu.pl

ORCID ID's: 0000-0001-7208-0482 (M. Mojżeszko), 0000-0003-1871-0249 (M. Setty)

(C) 2020 Authors. This is an open access publication, which can be used, distributed and reproduced in any medium according to the Creative Commons CC-BY 4.0 License requiring that the original work has been properly cited. 


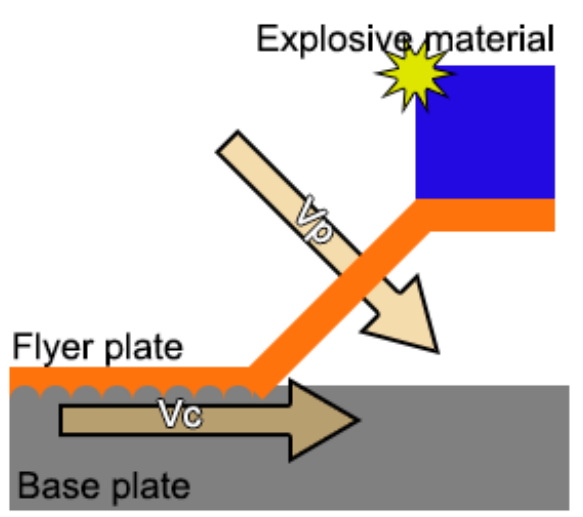

Fig. 1. Schematic concept of an explosive welding process: $V_{c}$ - velocity at the collision point, $V_{p}$ - impact velocity of a flyer plate

The explosive material is ignited from one edge; thus, forcing the flyer plate to bend at a specific angle that is a function of the initial distance between the plates, the so-called stand-off distance. If the impact angle and the velocity are within an acceptable range, the collision generates enough pressure and heat to weld the materials together successfully as mentioned by Fronczek (2017). Local generation of high temperature and pressure causes the melting of the material along the contact surface. The contact surface just before the collision is also cleaned by the plasma jet generated between the surfaces of the two colliding plates. Eventually, the liquified surfaces are joined and create a very characteristic shape of the interlayer along the contact area, as presented in Figure 2a.

As mentioned, the heat generation leading to a significant increase in the local temperature is one of the most critical factors in this process. During exposure to such elevated temperatures, materials undergo, e.g., phase transformation, recrystallization, or new intermetallic phases (Paul et al., 2013) are being created along the contact area, as presented in Figure 2b. As a result, significantly different local material properties are expected what is clearly visible from realized nanoindentation investigation presented in Figure 2c. In this case, the indentations from the vortex area show higher hardness than the initial materials of the flyer and base plates, respectively.

As described, explosive welding is an extremely sophisticated process involving various phenomena, which occur and interact in materials during a collision at high velocities and pressures. To properly understand different aspects of this process and extend experimental investigation capabilities, it is necessary to use numerical modeling. This approach can show some aspects of the process that are difficult, often even impossible to be captured experimentally. a) $\mathrm{Ti}$

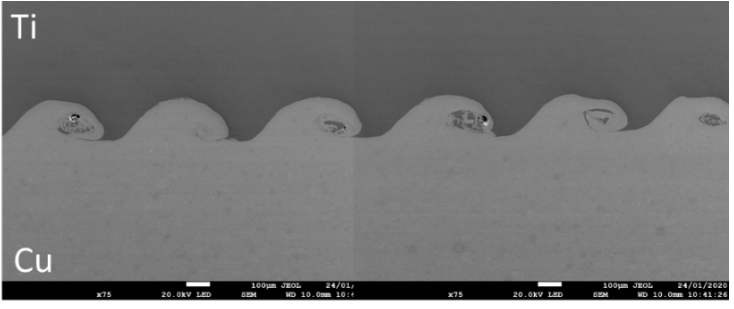

b)

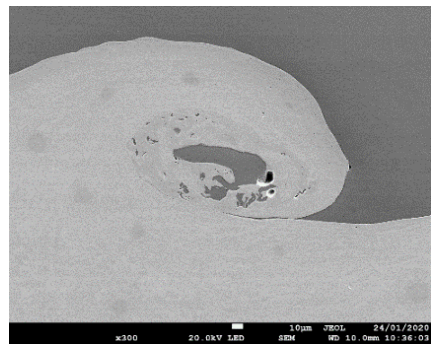

c)

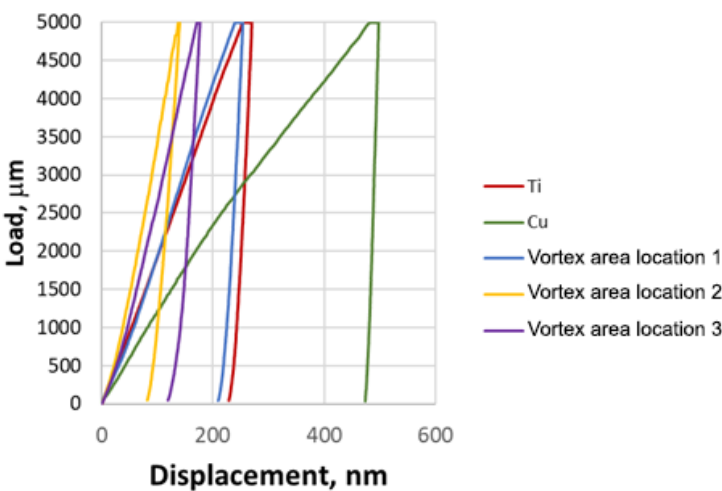

Fig. 2. Morphology of the interlayer along the contact area of the two plates after explosive welding (a), single vortex with visible intermetallic phases (b), nanoindentation measurement in the $\mathrm{Cu}, \mathrm{Ti}$, and three locations within the vortex area (c)

The most successful and reliable numerical approaches for simulations of dynamic processes are hybrid finite element methods (FEM), such as ALE (Arbitrary Lagrangian-Eulerian), which takes the strengths of the Lagrangian and Eulerian approaches and combines them to counter their limitations mentioned by Nassiri and Kinsley (2016). However, such complicated models are still ill-suited for problems involving extremely high speeds and deformations. The main issue is that the computational resources needed to conduct research are extremely high, additionally the computational time can also be affected by the model's complexity, which leads to greatly extended calculation times. Another drawback of the commonly applied mesh-based techniques is that high deformations can cause mesh degeneration, which is severe weakness of every model based on, e.g., FEM. Therefore, the authors decided to use a different method based on the SPH (Smoothed Particle Hydrodynamics) approach to study explosive welding. The $\mathrm{SPH}$ is a mesh-free method that recently proved to 
be an efficient solution in this research area (Li et al., 2017; Nassiri et al., 2017; Zhang et al., 2018; Bataev et al., 2019; Zhang \& Liu, 2019; Sun et al., 2020).

\section{Explosive welding simulation}

Compared to the standard FEM models, the SPH method does not use a connected grid of elements to discretize the computational domain. In the case of meshfree methods, the sample area is filled with particles which represent the physical volume of the sample material. Each particle has a limited influence over its neighbors, described by smoothing distance governed by the kernel function (Liu \& Liu, 2003). A Johnson-Cook (JC) (Madej et al., 2015) flow stress model was used in this work to describe the material behavior of colliding plates. The relationship between pressure, density, and internal energy was described by the Mie-Grüneisen equations of state (EoS) (Jinsong et al., 2020):

$$
\begin{aligned}
& P=P_{H}+\Gamma \rho\left(E-E_{H}\right) \\
& \Gamma \rho=\Gamma_{0} \rho_{0}=\text { constant } \\
& P_{H}=\frac{\rho_{0} c_{0} u(1+u)}{[1-(s-1) u]^{2}} \\
& E_{H}=\frac{1}{2} \frac{P_{H}}{\rho_{0}}\left(\frac{u}{1+u}\right)
\end{aligned}
$$

where: $E_{H}$ - energy at the reference state, $\Gamma$ - Grüneisen parameter, which represents a thermal pressure from a set of vibrating atoms, $\Gamma_{0}-$ Grüneisen coefficient, $\rho$ - current density, $\rho_{0}-$ initial density, $c_{0}$ - bulk sound speed, $s$ - coefficient of the slope of the shock-velocity function, $P$ - pressure, $u$ - specific volume. The subscripts " 0 " and " $H$ " denote that these parameters are either in the initial or Hugoniot state, respectively.

Material parameters for the EoS and flow stress models for the titanium and copper that were used in the simulation as a case study are collected in Table 1 (Wang et al., 2012).

The explosive material and the detonation process are not included explicitly in the final model to decrease computational times. Therefore, a fixed velocity was applied to the flyer plate to recreate the force resulting from the explosion and consider it in an implicit manner. During the research, three different detonation velocities were used $V_{d 1}=2000 \mathrm{~m} / \mathrm{s}, V_{d 2}=2500 \mathrm{~m} / \mathrm{s}$, $V_{d 3}=3000 \mathrm{~m} / \mathrm{s}$, that can be attributed to different explosive materials like Ammonium Nitrate Fuel Oil or ANFO. The resulting impact velocity for the investigated case studies are then calculated as:

$$
V_{p}=2 V_{d} \sin \frac{\beta}{2}
$$

where $\beta$ is the angle between plates.

Besides the explosion velocity, the initial impact angle is the second crucial parameter that can significantly change materials behavior during welding. The impact angle is related to the stand-off distance between the two plates that are used in the process setup. Two angles have been selected for the current investigation, namely $12^{\circ}$ and $16^{\circ}$.

Therefore, to investigate the influence of collision angle and detonation velocity, six numerical models (three detonation velocities and two initial impact angles) were developed, assembled, and executed. Figure 3 presents the initial states of the models for the two different impact angles. During the simulation, each of the subsequent models was based on the same material parameters and boundary conditions.

a)

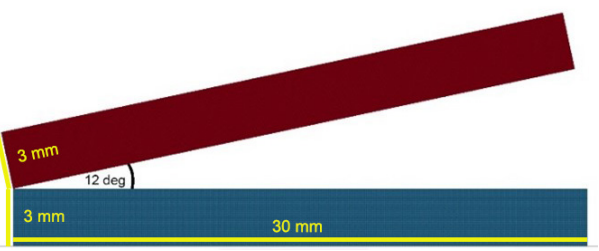

b)

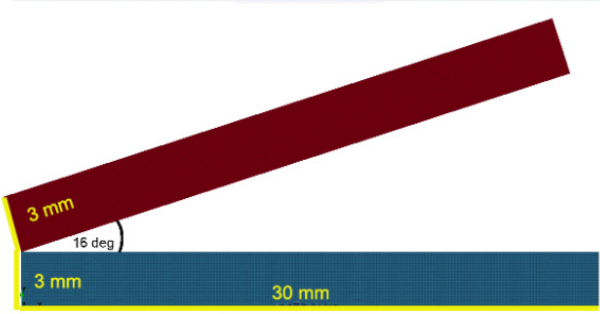

Fig. 3. Comparison of the two models developed for the current investigation with 12-degree (a) and 16-degree (b) initial impact angles

The first investigated case study was for the smallest detonation velocity of $2000 \mathrm{~m} / \mathrm{s}$. The obtained results for the two different collision angles are presented in Figure 4.

Table 1. Mie-Grüneisen EoS and Johnson-Cook flow stress model parameters for titanium and copper, respectively

\begin{tabular}{|l|c|c|c|c|c|c|c|c|c|c|}
\hline \multicolumn{1}{|c|}{ Material } & $\rho_{0}\left[\mathrm{~kg} / \mathrm{m}^{3}\right]$ & $\Gamma_{0}$ & $c_{0}[\mathrm{~m} / \mathrm{s}]$ & $s[-]$ & $u[\mathrm{GPa}]$ & $A$ & $B$ & $n$ & $m$ & $C$ \\
\hline Titanium & 4450 & 1.09 & 5220 & 0.767 & 43 & 1500 & 380 & 0.32 & 0.7 & 0.022 \\
\hline Copper & 8960 & 0.75 & 3940 & 1.489 & 98.7 & 70 & 292 & 0.31 & 1.09 & 0.025 \\
\hline
\end{tabular}


a)

b)
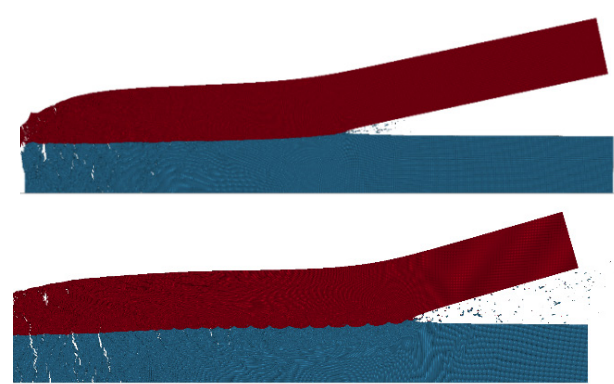

Fig. 4. Comparison of results obtained from the simulations with $2000 \mathrm{~m} / \mathrm{s}$ initial velocity of the flyer plate for 12-degree (a) and 16-degree (b) initial impact angles

As seen in Figure 4, the simulation resulted in a flat interface without clearly visible vortexes in both cases. The corresponding pressure and temperature distributions at the collision point are shown in Figure 5.
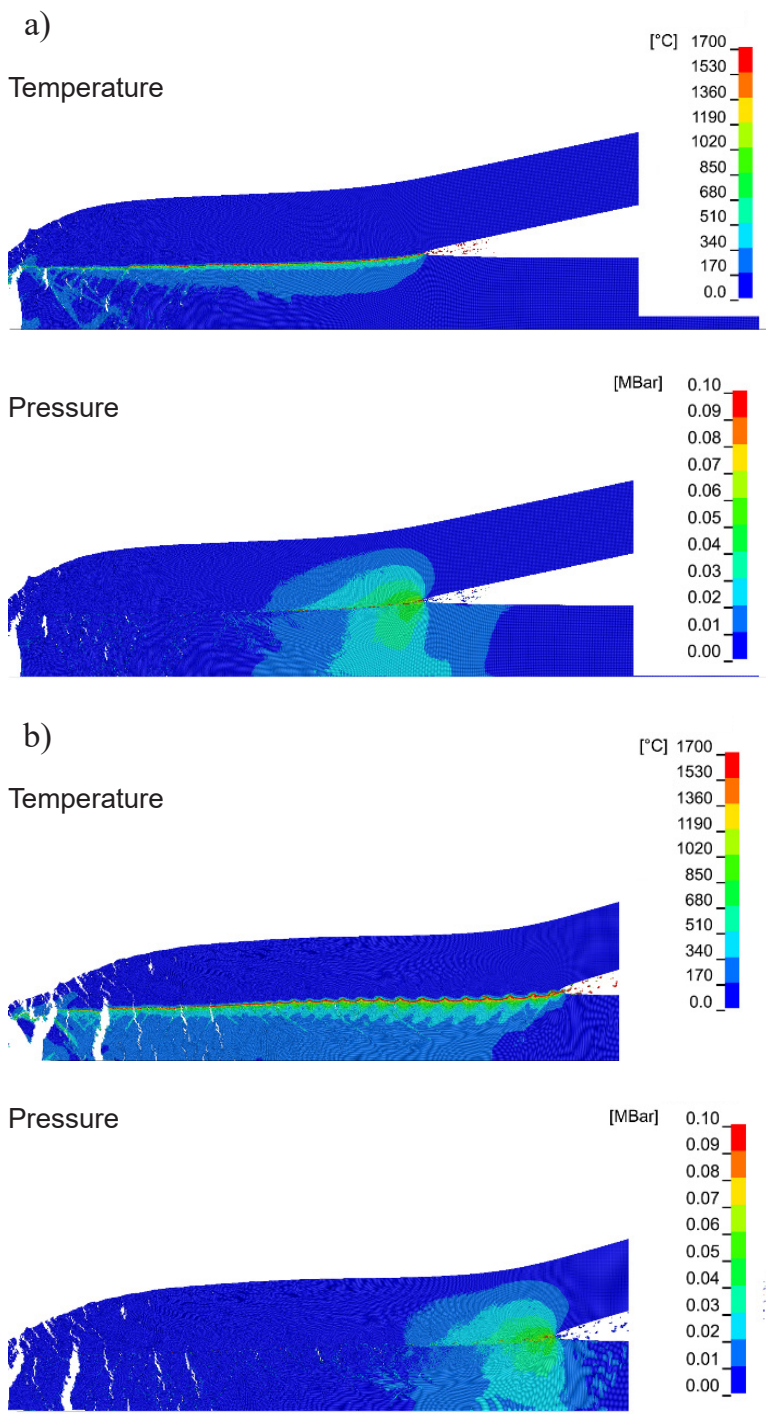

Fig. 5. Comparison of temperatures and pressures distribution from $2000 \mathrm{~m} / \mathrm{s}$ explosion velocity: 12-degree (a) and 16-degree (b) initial impact angles
As seen when the smaller detonation velocity is applied, especially for the low initial impact angle, the welding conditions may not be satisfied as the interface is quite flat and the local temperatures are generally too low to melt the material.

Therefore, the detonation velocity was increased to $2500 \mathrm{~m} / \mathrm{s}$. The comparison of results from 12- and 16-degree initial impact angles models is presented in Figure 6.
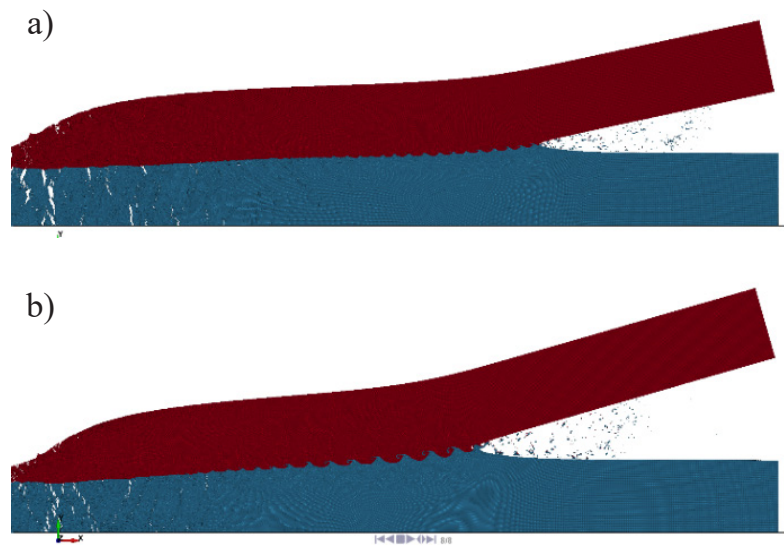

Fig. 6. Comparison of results from the model with $2500 \mathrm{~m} / \mathrm{s}$ initial velocity of the flyer plate: 12-degree (a) and 16-degree (b) initial impact angles

The visible geometrical differences between the interface features were measured as presented in Figure 7 in both vertical and horizontal directions.

a)

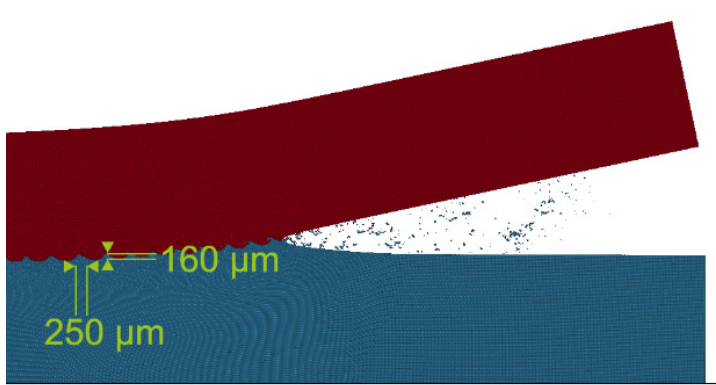

b)

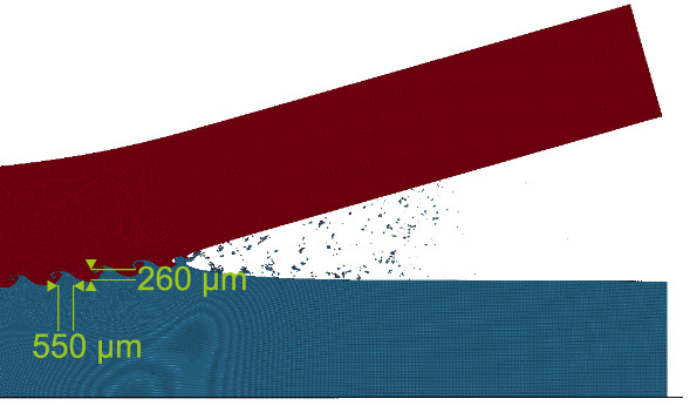

Fig. 7. Quantitative comparisons of interface geometrical aspects for 12-degree (a) and 16-degree (b) initial impact angles and initial velocity of $2500 \mathrm{~m} / \mathrm{s}$ 
The average length of the resulting vortexes in 12-degree model is approximately $250 \mu \mathrm{m}$ along the interface, while the calculated height $\sim 160 \mu \mathrm{m}$. In the case of 16-degree model, the average vortex length increases to $\sim 550 \mu \mathrm{m}$ with approx. $260 \mu \mathrm{m}$ in height. This agrees with the experimental results, which indicate that the higher the stand-off distance, the vortexes at the interface are better pronounced (Skuza et al., 2016). Additionally, both pressure and temperature fields are compared in Figure 8.

a)

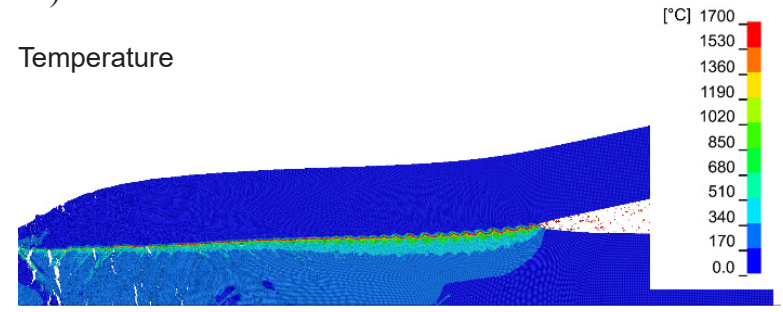

Pressure

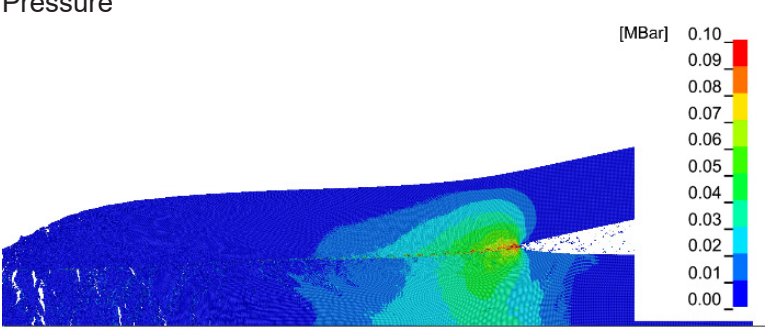

b)

Temperature

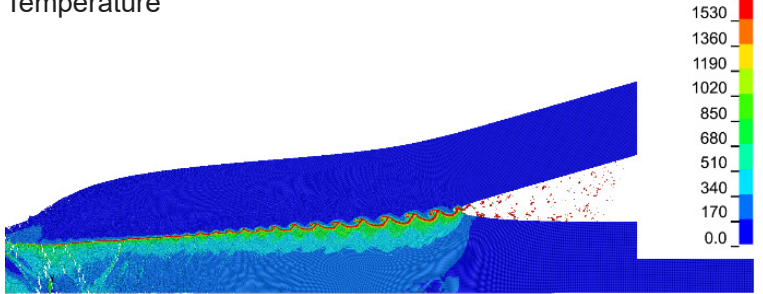

Pressure

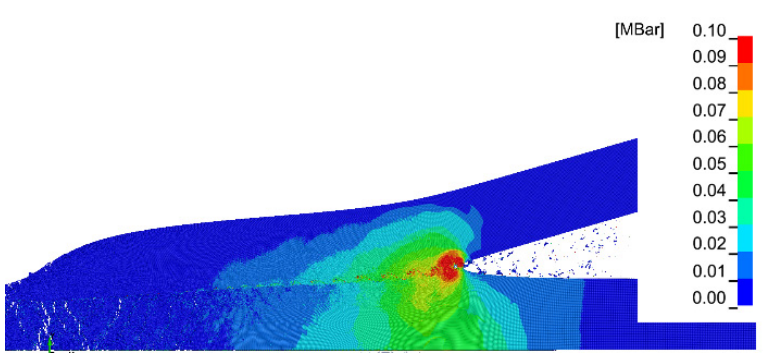

Fig. 8. Comparison of temperatures and pressures distribution from $2500 \mathrm{~m} / \mathrm{s}$ explosion velocity: 12-degree (a) and 16-degree (b) initial impact angles

As seen in the 16-degree case, the increase in the local temperature and pressure is higher, directly affecting the vortexes dimensions along the interface.
In the final set of simulations, the detonation velocity was increased to $3000 \mathrm{~m} / \mathrm{s}$, and the obtained results are presented in Figure 9.

a)

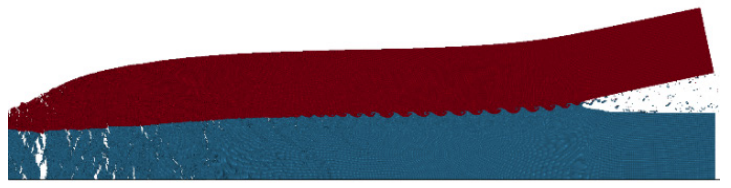

b)

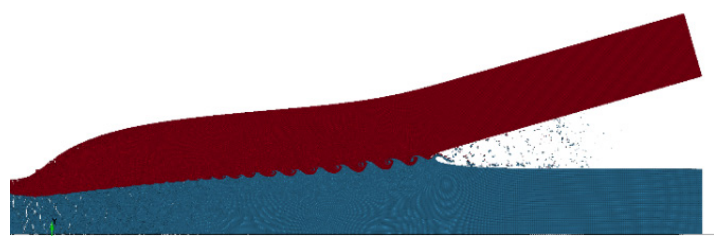

Fig. 9. Comparison of results from the model with $3000 \mathrm{~m} / \mathrm{s}$ initial velocity of the flyer plate: 12-degree (a) 16-degree (b) initial impact angles

This set of simulation results clearly shows how vital the collision velocity is for the welding process. As presented in Figure 10, in this case, the average length of the resulting vortexes in 12-degree model is approximately $500 \mu \mathrm{m}$ along the interface, while the calculated wave height is at the level of $\sim 260 \mu \mathrm{m}$. In the case of 16-degree model, the average vortex length is $\sim 900 \mu \mathrm{m}$ with approx. $500 \mu \mathrm{m}$ height.

a)

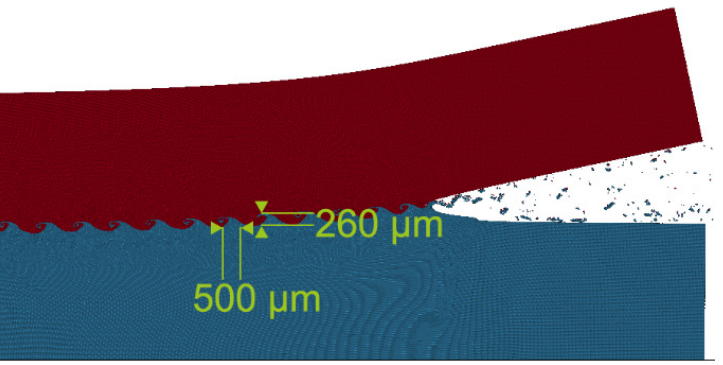

b)

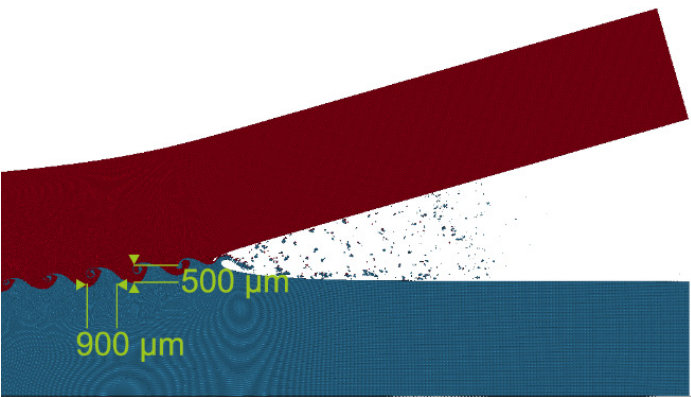

Fig. 10. Quantitative comparisons of interface geometrical aspects for 12-degree (a) and 16-degree (b) initial impact angles and initial velocity of $3000 \mathrm{~m} / \mathrm{s}$

This detonation velocity impact is also evident when the pressure and temperature fields are considered, as seen in Figure 11. 
a)

Temperature
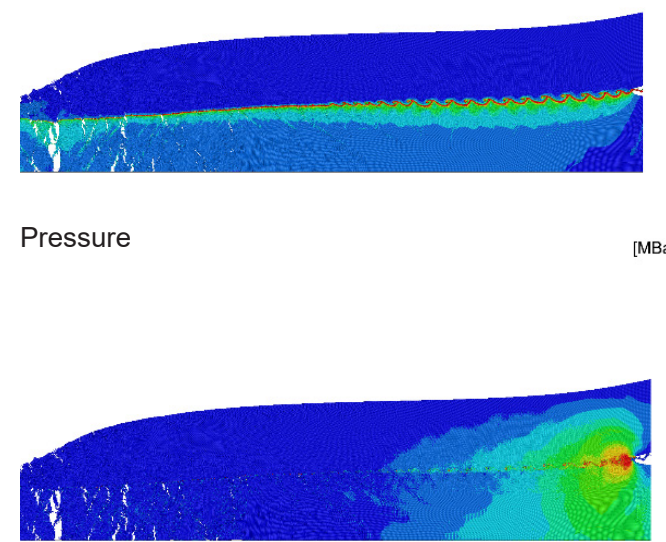

b)

Temperature
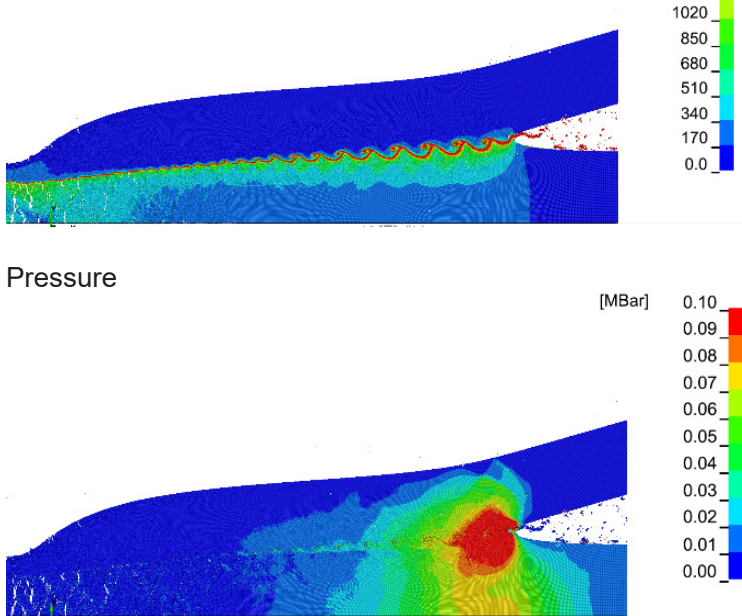

Fig. 11. Comparison of temperatures and pressures distribution from $3000 \mathrm{~m} / \mathrm{s}$ explosion velocity 12-degree (a) and 16-degree (b) initial impact angles

The quantitative comparison of the results and differences obtained are summarized in Figure 12.

As presented in Figure 11, the angle between plates significantly impacts the resulting interface. When compared, it is clearly distinguishable that a higher angle results in more pronounced waves along with the contact interface.

\section{QUANTITIVE ANALYSIS OF WAVE DIMENSIONS}

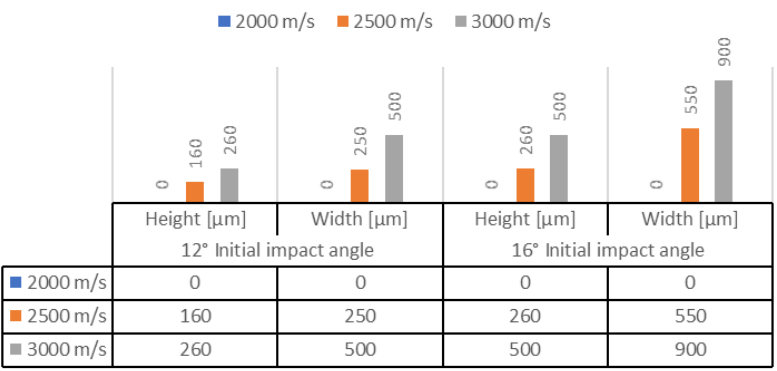

Fig. 12. Comparison of wave dimensions across all simulations

\section{Conclusions}

The conducted research has proven that different aspects of the process of explosive welding influence the interlayer's final morphology. During investigations, six different cases were considered and compared. Numerical simulations showed that both the initial angle between plates and the initial detonation velocity significantly impacted simulation results. A decrease in the angle between material plates resulted in a flattening of the vortexes along the interlayer, something which can affect welding quality. The flyer plate's initial velocity, associated with a type of explosive material, also heavily influences the process of interface creation leading to flat interlayer morphology. On the contrary with $500 \mathrm{~m} / \mathrm{s}$ increase in detonation velocity, the overall size of waves increased 2-times, leading to pronounced vortexes.

\section{Acknowledgment}

The work was realized as a part of fundamental research financed by the Ministry of Science and Higher Education, grant no. 16.16.110.663. Jiangting Wang's support with the SEM imaging is also gratefully acknowledged.

\section{References}

Akbari Mousavi, S.A.A., \& Farhadi Sartangi, P. (2009). Experimental investigation of explosive welding of cp-titanium/AISI 304 stainless steel. Materials \& Design, 30(3), 459-468.

Bataev, I.A., Tanaka, S., Zhou, Q., Lazurenko, D.V., Junior, A.M.J., Bataev, A.A., Hokamoto, K., Mori, A., \& Chen, P. (2019). Towards better understanding of explosive welding by combination of numerical simulation and experimental study. Materials and Design, 169, 107649.

Fronczek, D.M. (2017). Microstructural and kinetic characterization of the phenomena occurring at the clad's interfaces manufactured by explosive welding ( $\mathrm{PhD}$ thesis). IMMS PAS, Krakow. 
Jinsong, C., Wenjun, C., Shouan, C., Guiyu, Z., \& Tong, Z. (2020). Shock Hugoniot and Mie-Grüneisen EOS of TiAl alloy: A molecular dynamics approach. Computational Materials Science, 174, 109495.

Lednev, V.N., Sdvizhenskii, P.A., Ya Stavertiy, A., Ya Grishin, M., Tretyakov, R.S., Asyutin, R.D., \& Pershin, S.M. (2021). Online and in situ laser-induced breakdown spectroscopy for laser welding monitoring. Spectrochimica Acta Part B: Atomic Spectroscopy, 175, 106032.

Li, Y., Liu, C., Yu, H., Zhao, F., Wu, Z.L., Cuirong, L., Haibo, Y., Fei, Z., \& Zhisheng, W. (2017). Numerical simulation of Ti/A1 bimetal composite fabricated by explosive welding. Metals, 7(10), 407.

Li, Z., Beslin, E., den Bakker, A.J., Scamans, G., Danaie, M., Williams, C.A., \& Assadi, H. (2021). Bonding and microstructure evolution in electromagnetic pulse welding of hardenable Al alloys. Journal of Materials Processing Technology, 290, 116965.

Liu, G.R., \& Liu, M.B. (2003). Smoothed particle hydrodynamic: a meshfree particle method. World Scientific Publishing.

Madej, L., Perzynski, K., \& Paul, H. (2015). Numerical modelling of explosive welding on the basis of the coupled Eulerian Lagrangian approach. Key Engineering Materials, 651-653, 1415-1420.

Nassiri, A., \& Kinsey, B. (2016). Numerical studies on high-velocity impact welding: smoothed particle hydrodynamics (SPH) and arbitrary Lagrangian-Eulerian (ALE). Journal of Manufacturing Processes, 24(2), 376-381.

Nassiri, A., Zhang, S., Lee, T., Abke, T., Vivek, A., Kinsey, B., \& Daehn, G. (2017). Numerical investigation of CP-Ti \& Cu110 impact welding using smoothed particle hydrodynamics and arbitrary Lagrangian-Eulerian methods. Journal of Manufacturing Processes, 28(3), 558-564.

Paul, H., Lityńska-Dobrzyńska, L., \& Prażmowski, M. (2013). Microstructure and phase constitution near the interface of explosively welded aluminum/copper plates. Metallurgical and Materials Transactions A, 44(8), 3836-3851.

Skuza, W., Paul, H., Berent, K., Prazmowski, M., \& Bobrowski, P. (2016). Microstructure and mechanical properties of Ti/Cu clads manufactured by explosive bonding at different stand-off distances. Key Engineering Materials, 716, 464-471.

Sun, Z., Shi, Ch., Xu, F., Feng, K., Zhou Ch., \& Wu X. (2020). Detonation process analysis and interface morphology distribution of double vertical explosive welding by SPH 2D/3D numerical simulation and experiment. Materials and Design, 191, 108630.

Wachowski, M., Śnieżek, L., Szachogłuchowicz, I., Kosturek, R., \& Płociński, T. (2018). Microstructure and fatigue life of Cp-Ti/316L bimetallic joints obtained by means of explosive welding. Bulletin of the Polish Academy of Sciences, 66(6), 925-933.

Wang, X., Zheng, Y., Liu, H., Shen, Z., Hu, Y., Li, W., Gao, Y., \& Guo, C. (2012). Numerical study of the mechanism of explosive/impact welding using Smoothed Particle Hydrodynamics method. Materials \& Design, 35, 210-219,

Wolf, M., Werner, J., \& Drummer, D. (2020). Weld seam morphology and bond strength of infrared and vibration welded SLS parts of polyamide 12 as a function of the layer build-up direction and the welding process. Additive Manufacturing, 36, 101451.

Zhang, Z.L., \& Liu, M.B. (2019). Numerical studies on explosive welding with ANFO by using a density adaptive SPH method. Journal of Manufacturing Processes, 41, 208-220.

Zhang, Z.L., Feng, D.L., \& Liu, M.B. (2018). Investigation of explosive welding through whole process modeling using a density adaptive SPH method. Journal of Manufacturing Processes, 35, 169-189. 
